\title{
Hospital optimista: juntos podemos cambiar el mundo
}

\author{
María Josefina PelÁez \\ pelaezm@uji.es \\ MARISA SALANOVA SORIA \\ salanova@uji.es \\ ISABEL MARÍA MARTÍNEZ \\ imartine@uji.es
}

\section{Resumen}

Introducción: Este trabajo tiene como objetivo presentar el proyecto Premios Hospital Optimista desde un planteamiento científico centrado en la mejora del bienestar de las personas y las organizaciones. Su objetivo es contribuir a la mejora de la salud de los pacientes hospitalizados fomentando y reconociendo las mejores iniciativas o prácticas llevadas a cabo en el ámbito sanitario que contribuyan a la creación de un entorno optimista para el paciente y su familia. Método: Durante la gala se da a conocer el top ten de las candidaturas que participan de la iniciativa para cada categoría (centros, servicios, proyectos y proyectos pediátricos). Las dos primeras categorías toman como base el modelo de organización sanitaria saludable y resiliente (Healthy \& Resilient Organization) (Salanova, 2008, 2009; Salanova, Llorens, Cifre y Martínez, 2012). En este trabajo se presentan las líneas generales de estos premios, las diferentes categorías que participan y los criterios de participación, las metodologías de evaluación y los criterios de ponderación. Resultados: El mérito a las mejores prácticas, recursos y resultados organizacionales que desarrollaron los participantes de esta primera edición de premios (2015) fueron concedidos a 5 centros de atención primaria, 10 servicios/equipos multidisciplinares y 10 proyectos de actuación de todo el país. Conclusión: Este proyecto muestra un diseño innovador de investigación en el procedimiento y la recogida de datos, además de que utiliza una nueva forma de difundir los resultados de investigación a través de una gala de premios.

Palabras clave: organizaciones sanitarias saludables, psicología positiva, prácticas organizacionales, empleados saludables, resultados organizacionales.

\section{Abstract}

Introduction: The aim of this study is to present the project Hospital Optimista Awards based on a scientific approach focused on improving people's and organization's wellbeing. Its aim is to contribute to the improvement of hospitalized patient's health by encouraging and recognizing the best initiatives and/or practices carried out in the health sector that contribute to creating an optimistic environment for the patient and his/her family. Method: During the gala the Top Ten candidates that participate in each 
category (hospitals, services, adult projects and paediatric projects) are announced. For the two first categories the ranking is based on the HERO-HOS model (Healthy and Resilient Healthcare Organizations; Salanova, Llorens, Cifre y Martínez, 2012). In this paper, the outlines of these Hospital Optimista Awards, the different categories involved and the criteria for participation, assessment methodologies and evaluation criteria are presented. Results: The merit to the best practices, resources and organizational outcomes developed by the participants of this first awards (2015) edition were awarded to 5 primary care centres, 10 multidisciplinary services and 10 action projects across the country. Conclusion: This project shows an innovative design research in the procedure and data collection, as it implements a new way of disseminating research results through an awards gala.

Keywords: Healthy healthcare organizations, positive psychology, organizational practices, healthy employees, organizational outcomes.

\section{Introducción}

Las sociedades modernas se caracterizan por conllevar períodos de crisis y de cambios sociales y económicos constantes. Esta situación requiere de organizaciones modernas que se enfoquen en la salud de sus empleados, los equipos de trabajo y el contexto organizacional. La investigación ha mostrado la importancia de integrar programas de salud en las políticas organizacionales y culturales, dentro de los planes estratégicos de las organizaciones (Llorens, Salanova, Torrente y Acosta, 2013; Salanova, 2009; Salanova, Llorens, Cifre y Martínez, 2009).

De esta manera, si las organizaciones, además de buscar resultados excelentes, se esfuerzan por promocionar la salud psicosocial de sus trabajadores y buscan incrementar el capital psicológico positivo, nos encontramos ante una organización saludable y resiliente (Healthy \& Resilient Organization, HERO) siendo HERO-HOS (Healthy \& Resilient Organization - Hospitales) una adaptación de este modelo al ámbito sanitario (Salanova, 2008, 2009; Salanova, Llorens, Cifre y Martínez, 2012).

El concepto de HERO ha sido desarrollado por el equipo WANT, Prevención Psicosocial y Organizaciones Saludables ${ }^{1}$ de la Universitat Jaume I de Castellón, a través de su investigación y publicaciones científicas. Este concepto se enmarca en la disciplina de la psicología de la salud ocupacional y sus más recientes avances en la psicología de la salud ocupacional positiva (PSOP) y en última instancia en la psicología positiva. Se trata de un modelo heurístico y teórico que integra evidencia proveniente de las investigaciones sobre estrés laboral, dirección de recursos humanos, comportamiento organizacional y PSOP (Salanova y cols., 2009).

De manera genérica, una HERO es considerada como una organización que lleva a cabo esfuerzos y acciones sistemáticas, planificadas y proactivas para mejorar la salud, los procesos y los resultados de los empleados y la organización. Esos esfuerzos suponen implementar buenas prácticas organizacionales relacionadas con la promoción y optimización de recursos relacionados con la mejora de las tareas (por ejemplo, autonomía), el ambiente social de la organización (por ejemplo, liderazgo transformacional y saludable) y la organización (por

1. Anteriormente se llamaba WONT (Work \& Organization NetWork); ahora pasa a llamarse WANT por su significado en inglés. 
ejemplo, estrategias de conciliación trabajo / vida privada). Estos aspectos se integran en tres grandes componentes interrelacionados: (1) recursos y prácticas organizacionales saludables, (2) empleados y grupos de trabajo saludables que gozan de bienestar psicosocial con la presencia de recursos personales y (3) resultados organizacionales saludables (Salanova, 2008, 2009; Salanova y cols., 2012).

Específicamente en el contexto sanitario, se considera una organización sanitaria saludable y resiliente (Healthy \& Resilient Organization - Hospital) (HERO-HOS) como aquella que se esfuerza por garantizar la calidad de vida laboral de sus empleados, sobrevivir y prosperar en contextos de cambio económico y social, con la capacidad de crear ambientes de trabajo que contribuyan a mejorar la práctica profesional y la salud de los profesionales sanitarios y, en definitiva, a mejorar la calidad del servicio prestado al paciente/familiar (Hernández-Vargas, Llorens-Gumbau y Rodríguez-Sánchez, 2014; Matos, Neu-shotz, Griffin y Fitzpatrick, 2010; McAllister y Lowe, 2011; Salanova, Rodríguez-Sánchez, del Líbano y Ventura, 2012).

Para integrar estos tres componentes del modelo, la investigación realizada desde el equipo WANT de la Universitat Jaume I proporciona evidencia sobre el efecto positivo que los recursos y las prácticas organizacionales saludables tienen sobre el bienestar de los empleados (por ejemplo, eficacia colectiva, work engagement y resiliencia) y que, a su vez, tienen un impacto positivo sobre los resultados saludables (por ejemplo, desempeño evaluado por el propio supervisor) (Acosta, Salanova y Llorens, 2012; Salanova y cols., 2012). Específicamente en el contexto de las organizaciones sanitarias, se han llevado a cabo diversos estudios desde el equipo que ponen a prueba relaciones específicas entre las variables de los componentes del modelo HERO a partir de metodologías cuantitativas y cualitativas.

Con respecto a los estudios cuantitativos, numerosas investigaciones ponen de manifiesto la importancia de considerar variables psicosociales para explicar los resultados del trabajo ya sea sobre los trabajadores o sobre los resultados organizacionales. En una investigación llevada a cabo en el personal sanitario de un hospital de la Comunidad Valenciana se mostró cómo aquellos empleados con elevados niveles de creencias de eficacia presentan mayor afecto positivo y más engagement en el trabajo que, a su vez, se relaciona positivamente con mayor calidad de servicio (desempeño, compromiso y calidad percibida) (HernándezVargas y cols., 2014). En la misma línea se evidenció el papel de las creencias de autoeficacia de enfermeras de cuidados intensivos en la predicción de prácticas de colaboración futuras y se confirmó el papel mediador del compromiso grupal en esta relación (Le Blanc, Schaufeli, Salanova, Llorens y Nap, 2010). En otro estudio se mostró cómo la autoeficacia y el engagement en el trabajo de las enfermeras son los mecanismos psicológicos mediadores que explican la relación entre el liderazgo transformacional (de sus jefas) y su desempeño extra-rol (Salanova, Lorente, Chambel y Martínez, 2011). Los resultados de otros estudios apoyan estos datos sobre la importancia de los recursos interpersonales en el trabajo (por ejemplo, buena cooperación con compañeros), los cuales amortiguan los efectos negativos de la disonancia emocional en el desempeño laboral en un estudio longitudinal de 4 años, mientras que el rol de los recursos personales (por ejemplo, optimismo) parece ser menos importante para mantener un alto desempeño laboral bajo condiciones de disonancia emocional (RodríguezSánchez, Hakanen, Perhoniemi y Salanova, 2013). En el ámbito de las intervenciones, se ha constatado el efecto de un taller de trabajo sobre inteligencia emocional en enfermeras de un hospital de la Comunidad Valenciana (Rodríguez, Llorens y Salanova, 2006). Los resultados obtenidos muestran incrementos, tras la realización del taller, en competencias emocionales que podían influir en la disminución del sufrimiento empático.

Complementariamente a estos resultados obtenidos mediante metodología cuantitativa, se han mostrado evidencias sobre evaluación de las percepciones de organización saludable (Salanova y cols., 2011); el análisis de prácticas organizacionales saludables y los resultados orga- 
nizacionales saludables (Salanova y cols., 2012); el análisis de las frecuencias de las prácticas organizacionales saludables en pequeñas y medianas empresas (pymes) (Acosta, y cols., 2012) y el análisis del significado del concepto de organización saludable y sus elementos clave a través de análisis de contenido (Acosta, Cruz, Salanova y Llorens, 2015), todo ello utilizando metodología cualitativa. Específicamente en el contexto sanitario, a través de un estudio de casos en hospitales, se puso en evidencia que las principales prácticas organizacionales saludables en estas organizaciones sanitarias fueron la gestión del impacto ambiental y la comunicación e información organizacional (Cifre, Acosta y Colombo, 2011; Acosta y cols., 2012).

Con el objetivo de integrar los estudios basados en el modelo HERO en el ámbito sanitario, este estudio pretende presentar los aspectos generales del proyecto Premios Hospital Optimista, que en sus categorías de centros y servicios reconocen las mejores iniciativas o prácticas llevadas a cabo en el ámbito sanitario, basándonos en el modelo HERO y sus tres componentes. El objetivo de estos premios es contribuir a la mejora de la salud de los pacientes hospitalizados, sus acompañantes o familiares y los propios trabajadores, fomentando y reconociendo las iniciativas que contribuyan a la creación de un entorno positivo y optimista para el paciente y su familia.

\section{Método}

\section{Bases de la convocatoria}

Los Premios Hospital Optimista se crearon en el año 2015 y constituyen una iniciativa privada sin ánimo de lucro. La idea surgió de Vygon y la organización de estos premios corre a cargo, además de Vygon, del equipo de investigación WANT de la Universitat Jaume I, la Fundación Grupo OTP y la Fundación Atresmedia. Todos ellos unidos con un objetivo común, que es contribuir a la mejora de la salud de los pacientes hospitalizados, fomentando y reconociendo, a través de una gala anual, las mejores iniciativas o prácticas llevadas a cabo en el ámbito sanitario, con el objetivo de fomentar la excelencia organizacional y dar a conocer y replicar las mejores iniciativas en el país. En cada categoría se da a conocer el top ten de las candidaturas que están invitadas a participar en la gala, a las cuales se las considera como organizaciones sanitarias saludables y resilientes (Salanova y cols., 2012). Se premian a las tres mejores con un trofeo conmemorativo y un premio en metálico de una cantidad de al menos $1000 €$, dinero que es destinado a una ONG de su elección, a la cual desea ayudar a través de la entrega del importe.

Durante el periodo de inscripción, los hospitales, centros, servicios y representantes de proyectos de todo el país que deseen participar envían su candidatura. La participación en los premios es gratuita y el nivel mínimo de participación varía en función del número de empleados de la organización sanitaria. Cuanto más alta sea la participación, mayor fiabilidad tienen los resultados, y los resultados más fiables obtendrán mejor nota. Los datos proporcionados por los participantes son anónimos o confidenciales. Solo se dan a conocer los nombres de los diez mejores en cada categoría.

\section{Categorías, criterios de participación y medidas de evaluación}

Estos premios cuentan con cuatro categorías: (1) categoría hospitales y centros de atención primaria; (2) categoría servicios/equipos multidisciplinares; (3) categoría proyectos llevados a cabo por hospitales, ONG, fundaciones o empresas en los centros sanitarios y (4) cate- 
goría proyectos pediátricos llevados a cabo por hospitales, ONG, fundaciones o empresas en los centros sanitarios.

Para las dos primeras categorías (centros y servicios), se ofrece la realización, sin coste, de un diagnóstico de salud y bienestar organizacional del equipo o del hospital. Se premian los hospitales y servicios con los mejores entornos psicosociales. La participación en esta modalidad de premios implica contestar un cuestionario online por parte de un número significativo de miembros del centro hospitalario. Consiste en una evaluación científico-aplicada reconocida por revistas e instituciones de impacto internacional: el cuestionario HERO-HOS del equipo de investigación WANT de la Universitat Jaume I, que lleva entre 15 y 20 minutos de rellenar, y evalúa, en 87 ítems, sus tres componentes relacionados con el entorno de trabajo: (1) recursos y prácticas organizacionales saludables (por ejemplo, estilo de liderazgo, autonomía, clima de apoyo social, nivel de exigencia respecto a la empatía); (2) empleados saludables, refiriéndose al nivel de bienestar psicológico de los profesionales (por ejemplo, optimismo, creencias de eficacia, engagement, confianza vertical, confianza horizontal, resiliencia); y (3) resultados organizacionales saludables (por ejemplo, calidad de servicio sanitario, desempeño intra y extra rol). Se responden a estas dimensiones utilizando una escala Likert de 7 puntos de anclaje que oscila de 0 (totalmente en desacuerdo / nunca) a 6 (totalmente de acuerdo / siempre). Un ejemplo de las preguntas respecto a la escala de clima de apoyo social del componente recursos y prácticas organizacionales es: «El supervisor/a manifiesta su preocupación por nuestros problemas personales».

Respecto a las dos últimas categorías, proyectos y proyectos pediátricos, se premian las mejores iniciativas y proyectos cuyos objetivos sean la promoción de la salud psicosocial del/ la paciente y de sus familiares. El criterio de participación en estas categorías implica enviar dos documentos: una hoja de inscripción cumplimentada y una memoria explicativa, en la que se especifiquen los objetivos del proyecto y su relación con el plan estratégico de la organización, los antecedentes, la metodología, el plan de trabajo, las aportaciones del proyecto y la medición del mismo.

\section{Criterios de selección y análisis de los datos}

Con respecto a los criterios de selección de los trabajos para las categorías centros y servicios, es decir, para valorar si están dentro del top ten de los mejor evaluados, el ganador será aquel centro sanitario o servicio que obtenga mayores indicadores en los criterios de medida utilizados comparados con el resto de centros sanitarios y servicios participantes.

Para valorar los resultados y elegir a los ganadores, se utiliza el siguiente criterio de ponderación para las categorías centros y servicios: para la categoría centros, el $65 \%$ corresponde a los resultados del cuestionario, el $25 \%$ al nivel de participación y el $10 \%$ restante a una entrevista y checklist realizados a la dirección del centro sanitario o a un representante de la organización; para la categoría servicios, el $75 \%$ del total es atribuido a los resultados del cuestionario y el $25 \%$ restante al nivel de participación de los trabajadores. Con respecto a la ponderación interna del cuestionario, a cada variable se le asignó un porcentaje dependiendo de la relevancia para este estudio (por ejemplo, $10 \%$ a la media de optimismo, $10 \%$ a la media de prácticas, $4,5 \%$ a la media de autonomía para la categoría servicios).

Para el análisis de los cuestionarios HERO-HOS de las categorías centros y servicios, se obtiene, a partir de los resultados, la media general de cada variable y su desviación estándar, la cual es comparada con las demás medias para obtener el ranking final. Las entrevistas realizadas a los centros son analizadas mediante análisis de contenido (Ahuvia, 2001). Esta 
técnica se utiliza para analizar categorías y obtener conclusiones basadas en un marco teórico previo (Denecke y Nejdl, 2009).

Para las categorías proyectos y proyectos pediátricos, el procedimiento de selección está organizado en dos fases. En la primera, los proyectos recibidos según las normas de los premios son puntuados por los organizadores según los criterios siguientes: (1) alcance, es decir, número de pacientes y familiares que se benefician de la medida; (2) impacto, esto es, en qué medida este proyecto genera bienestar; (3) medición, esto es, si se miden los resultados obtenidos y (4) originalidad, es decir, en qué medida este proyecto es novedoso. En la segunda fase, los 25 mejores proyectos según los criterios anteriores pasan a ser evaluados y votados por un jurado compuesto por personalidades relevantes en los ámbitos de la medicina, el management, la medicina del trabajo, la psicología positiva y el derecho del trabajo.

\section{Resultados}

Se comentarán a continuación los resultados de los top ten que participaron en la primera edición de los Premios Hospital Optimista (2015). Se inscribieron a estos premios un total de 130 hospitales, entre ellos 29 servicios, 13 hospitales y centros de atención primaria y 94 proyectos. Los que participaron de la evaluación mediante cuestionarios fueron 771 trabajadores pertenecientes a 5 hospitales y centros de atención primaria y 18 servicios/equipos multidisciplinares. El porcentaje de participación para las categorías centros fue de $57,3 \%$, mientras que para la categoría servicios, fue del 70,6\%.

En la primera gala de estos premios, llevada a cabo el 28 de abril del 2015 en Valencia, se dio a conocer el resultado de los top ten de las distintas categorías que participaron. Con respecto a las categorías centros y servicios, los resultados obtenidos mediante los criterios de evaluación, llevaron a establecer el ranking top cinco de las mejores candidaturas que participaron en la categoría centros y el top ten de la categoría servicios. En las figuras 1 y 2 se presentan las medias de las variables del resultado final, para las categorías centros y servicios respectivamente.

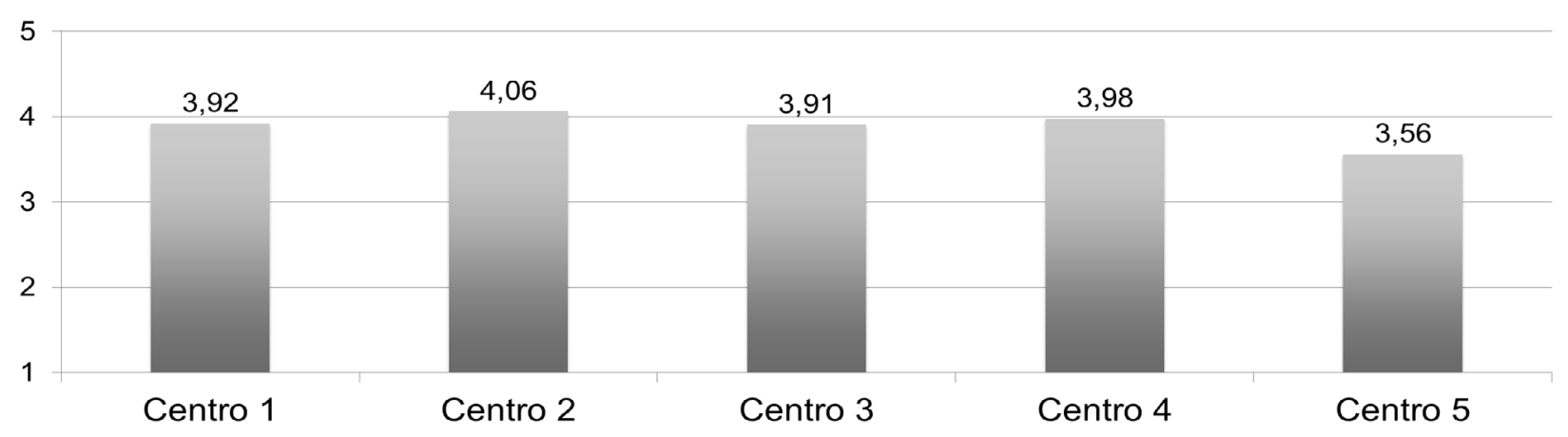

Figura 1. Medias de los resultados HERO-HOS (categoría centros) 


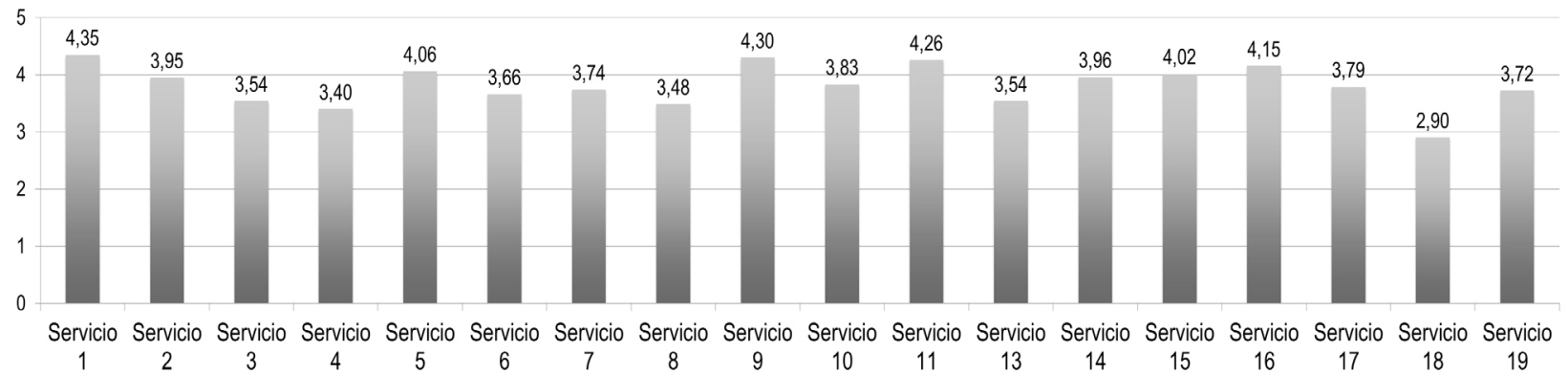

Figura 2. Medias de los resultados HERO-HOS (categoría servicios)

Respecto a la categoría proyectos llevados a cabo por hospitales, ONG, fundaciones o empresas en los centros sanitarios, se presenta a continuación el top ten de las mejores candidaturas:

- Proyecto HU-Cl para la humanización de los cuidados intensivos: este proyecto busca promover una medicina más próxima al paciente y más respetuosa con sus necesidades y deseos.

- Proyecto 12 meses, 12 gestos: se trata de un programa de competencias profesionales que fomenta la atención cercana y humana. El principal objetivo del proyecto es humanizar los cuidados y lograr que tecnicismo y humanismo vayan de la mano.

- Proyecto Entorno más amable: cuidamos de ti, tú cuida de ellos. Se trata de un programa de humanización a la atención a los cuidadores de familiares con enfermedad crónica y estancia prolongada. Una de las acciones llevadas a cabo fue realizar un gimnasio para los cuidadores.

- Proyecto CuidART: es un proyecto integral de arte que se lleva a cabo en todo el hospital.

- Proyecto El sonido de la vida: programa de musicoterapia en paliativos pediátricos.

- Proyecto EmocionARTE: se trata de un amplio programa de actividades musicales y plásticas para el desarrollo emocional en aulas hospitalarias con evaluación posterior.

- Proyecto ¿Dónde te acompaño?: es un servicio de taxistas voluntarios que trasladan a pacientes y sus familias sin recursos en sus días libres.

- Proyecto Voluntariado y talleres informativos: se trata de un programa integral de servicios y formación a pacientes, familiares y personal sanitario. El programa consiste en un servicio de voluntariado que ofrece actividades a nivel asistencial y talleres de psicooncología informativos a nivel médico y psicológico para fomentar la parte activa del paciente en su recuperación.

- Proyecto Haciendo sonreír a los niños: es un taller para decorar espacios infantiles del hospital con materiales reciclados del área quirúrgica. La decoración es elaborada por usuarios/as de la comunidad terapéutica de salud mental en el taller color y taller del reciclado.

- Proyecto Efectos del yoga y la meditación: este proyecto es parte de un estudio para probar los efectos del yoga y la meditación sobre el estrés de los profesionales sanitarios de unidades de cuidados intensivos. 
Con respecto a la cuarta y última categoría, la de proyectos pediátricos llevados a cabo por hospitales, ONG, fundaciones o empresas en los centros sanitarios, se realiza una mención especial en la gala a la mejor candidatura: el proyecto de estudio de la ansiolisis preoperatoria, una terapia combinada de fármacos con intervención de payasos de un hospital.

\section{Conclusiones}

El objetivo de este trabajo era presentar el proyecto Premios Hospital Optimista, con datos de la primera gala, cuya misión es contribuir a la mejora de la salud de los pacientes hospitalizados fomentando y reconociendo las iniciativas que contribuyan a la creación de un entorno optimista para el paciente y su familia.

Este proyecto muestra un diseño innovador de investigación en el procedimiento y la recogida de datos, además de que utiliza una nueva forma de difundir los resultados de investigación a través de una gala de premios. A nivel práctico, pone de manifiesto el esfuerzo que se hace desde los centros y los servicios de salud, quienes ponen en valor lo que hacen para mejorar su entorno haciendo públicas sus iniciativas y a quienes se les reconoce a través de estos premios como organizaciones sanitarias saludables y resilientes (HERO-HOS). Así es como, al hacer públicas estas iniciativas a nivel nacional, las organizaciones dan a conocer su trabajo interna y externamente y, de esta manera, sirven de espirales virtuosas y contagio de positividad y optimismo a otros centros y servicios del ámbito de la salud.

Por medio de la participación en la gala, los servicios y los centros, al obtener de forma gratuita una evaluación científica del nivel de eficacia de sus equipos de trabajo, acceden a conocer su nivel actual y, a partir del mismo, pueden emprender acciones de mejora y evaluación en el tiempo.

A nivel teórico, los resultados obtenidos apoyan las evidencias proporcionadas por la investigación previa realizada por el equipo WANT en el contexto de las organizaciones sanitarias, sobre las relaciones específicas entre las variables de los componentes del modelo HERO, es decir, el efecto positivo que los recursos y las prácticas organizacionales saludables tienen sobre el bienestar de los empleados y que, a su vez, tienen un impacto positivo sobre los resultados saludables (Acosta, Salanova y Llorens, 2012; Salanova y cols., 2012), constatando la interrelación de los tres grupos de variables.

Una limitación de este trabajo es que, al estar acotado el objetivo a presentar las líneas generales de estos premios, no nos hemos centrado en realizar análisis estadísticos que muestren las relaciones y la incidencia entre las variables de los componentes que se han evaluado. Sin embargo, estos estudios están siendo realizados actualmente por el equipo WANT. Así mismo, para futuras investigaciones, sería interesante realizar análisis comparativos de los resultados del cuestionario HERO-HOS con otros estudios realizados a organizaciones sanitarias evaluadas con la misma metodología.

Por otro lado, el análisis se ha centrado en un estudio transversal de los resultados de la primera gala de estos premios (2015). Es por ello que futuros estudios podrían comparar resultados de esta primera edición con los de próximas ediciones mediante un diseño longitudinal con metodología cuantitativa y cualitativa que permita realizar análisis de las dinámicas temporales de los resultados. 


\section{Referencias bibliográficas}

Acosta, H., Cruz-Ortíz, V. M., Salanova, M. y Llorens, S. (2015). Organizaciones saludables: Analizando su significado desde el modelo HERO. Revista de Psicología Social, 30, 323350.

Acosta, H., Salanova, S. y Llorens, S. (2012). ¿Qué prácticas organizacionales saludables son más frecuentes en las empresas? Un análisis cualitativo. Fórum de recerca, 16, 1-16.

Ahuvia, A. (2001). Traditional, interpretive, and reception based content analyses: Improving the ability of content analysis to address issues of pragmatic and theoretical concern. Social Indicators Research, 54, 139-172.

Cifre, E., Acosta, H. y Colombo, V. (2011). Resultados de la evaluación cualitativa: Entrevistas a dirección. En S. Llorens y M. Salanova (eds.), Organizaciones sanitarias saludables y resilientes: Un estudio de caso en hospitales (pp. 63-67). Castellón: Fundación DávalosFletcher.

Bustamate, M., Llorens, S. y Acosta, H. (2014). Empatía y calidad de servicio: El papel clave de las emociones positivas en equipos de trabajo. Revista Latinoamericana de Psicología Positiva, 1, 7-17.

Denecke, K. y Nejdl, W. (2009). How valuable is medical social media data? Content analysis of the medical web. Information Sciences, 179, 1870-1880.

Hernández-Vargas, C. I., Llorens-Gumbau, S. y Rodríguez-Sánchez, A. M. (2014). Empleados saludables y calidad de servicio en el sector sanitario. Anales de Psicología, 30, 247-258.

Le Blanc, P. M., Schaufeli, W. B., Salanova, M., Llorens, S. y Nap, R. E. (2010). Efficacy beliefs predict collaborative practice among intensive care unit nurses. Journal of advanced nursing, 66, 583-594.

Llorens, S., Salanova, M., Torrente, P. y Acosta, H. (2013). Interventions to promote Healthy \& Resilient Organizations (HERO) from Positive Psychology. En G. F. Bauer y G. J. Jenny (eds.), Salutogenic organizations and change: The concepts behind organizational health intervention research (pp. 91-106). Zurich: Springer.

McAllister, M. y Lowe, J. B. (2011). The resilient nurse: Empowering your practice. New York: Springer Publishing Co.

Matos, P. S., Neushotz, L. A., Griffin, M. y Fitzpatrick, J. J. (2010). An exploratory study of resilience and job satisfaction among psychiatric nurses working in inpatient units. International Journal of Mental Health Nursing, 19, 307-312.

Rodríguez-Sánchez, A., Hakanen, J. J., Perhoniemi, R. y Salanova, M. (2013). With a little help from my assistant: buffering the negative effects of emotional dissonance on dentist performance. Community Dentristy \& Oral Epidemiology, 41, 415-423.

Rodríguez-Sánchez, A., Llorens, S. y Salanova, M. (2006). Taller de trabajo sobre inteligencia emocional en enfermeras: eficacia a corto plazo. Gestión Práctica de Riesgos Laborales, $29,46-51$.

Salanova, M. (2008). Organizaciones saludables y desarrollo de recursos humanos. Estudios Financieros, 303, 179-214.

Salanova, M. (2009). Organizaciones saludables, organizaciones resilientes. Gestión práctica de riesgos laborales, 58, 18-23.

Salanova, M., Cifre, E., Martínez, I. M., Llorens, S. y Lorente, L. (2011). Psychosocial risks and positive factors among construction workers. En S. Clarke, C. Cooper y R. Burke (eds.), Occupational health and safety: Psychological and behavioural challenges (pp. 295-320). Farnham, England: Gower. 
Salanova, M., Llorens, S., Cifre, E. y Martínez, I. M. (2009). Psicología de la Salud Ocupacional: State of the art. En M. Salanova (ed.), Psicología de la Salud Ocupacional (pp. 101113). Madrid: Editorial Síntesis.

Salanova, M., Llorens, S. y Rodríguez, A (2009). Hacia una psicología de la salud ocupacional más positiva. En M. Salanova (ed.), Psicología de la Salud Ocupacional (pp. 247-284). Madrid: Editorial Síntesis.

Salanova, M., Llorens, S., Cifre, E. y Martinez, I. M. (2012). We Need a Hero! Towards a Validation of the Healthy \& Resilient Organization (HERO) Model. Group Organization Management, 37, 785-822

Salanova, M., Llorens, S., Torrente, P. y Acosta, H. (2013). Intervenciones positivas para promover organizaciones saludables y resilientes. En F. Palací y M. Bernabé (eds.), Consultoría Organizacional (pp. 137-166). Madrid: Sanz y Torres.

Salanova, M., Lorente, L., Chambel, M. J. y Martínez, I. M. (2011). Linking transformational leadership to nurses' extra-role performance: the mediating role of self-efficacy and work engagement. Journal of Advanced Nursing, 67, 2256-2266.

Salanova, M., Rodríguez-Sánchez, A. M., Del Líbano, M. y Ventura, M. (2012). Organizaciones Sanitarias Saludables y Resilientes: Marco teórico y conceptual. En S. Llorens y M. Salanova (dirs.), Organizaciones sanitarias saludables y resilientes: Un estudio de caso en hospitales de Castellón y provincia. Castellón: Fundación Dávalos-Fletcher. 